\section{Immuntherapie früh beginnen}

E in Patient mit allergischem Asthma sollte so früh wie möglich hyposensibilisiert werden. Diese Forderung ergibt sich auch vor dem Hintergrund der molekularen Vorgänge dieser Erkrankung. Denn nur in der „allergischen“ Phase entfaltet die Immuntherapie durch ihren TH2/TH1-Switch ihre volle Wirksamkeit.

Ein guter Marker für die allergische Entzündungsreaktion beim Asthma bronchiale, so Dr. Virchow (Freiburg) sind die eosinophilen Granulozyten im Sputum. Ihre Konzentration korreliert mit Symptomen wie Atemwegsobstruktion, bronchiale Hyperreagibilität oder Medikamentenverbrauch. Eosinophile werden nach Allergenkontakt von aus TH2-Zellen sezernierten Interleukinen wie IL-5 und IL-13 in die Bronchien "gelockt" und verlängern dort das Überleben der Entzündungszellen. Dies ist der entscheidende Mechanismus der allergischen Spätreaktion, der letztlich für die Chronifizierung des Asthmas verantwortlich ist.

Virchow: „Bei sich wiederholendem Allergenkontakt wird dann langfristig ein sich verselbstständigender, chronischer Entzündungsweg eingeschlagen, bei dem der Allergenkontakt kaum mehr eine Rolle spielt und sich ein Asthmaanfall auch durch unspezifische Triggerfaktoren auslösen lässt.“ In diesem fortgeschrittenen Stadium scheinen die TH2-Zellen keinen großen Einfluss mehr auf die Erkrankung zu haben - jedenfalls sind bei diesen Patienten kaum mehr deren Leitzytokine IL-5 oder IL-13 nachweisbar.

So erscheint es auch vor dem Hintergrund dieser pathophysiologischen Grundlagen sinnvoll, mit einer Immuntherapie die interleukinabhängige Entzündungsreaktion zu einem möglichst frühen Zeitpunkt zu blockieren.

\section{Virchow C.}

Pneumologie-Symposium „Allergie und Asthma“, veranstaltet von der ALKSCHERAX Arzneimittel GmbH, Dresden 2000.

\section{Antihistaminerg, antientzündlich, antiobstruktiv}

\author{
Desloratadin, Nachfolgeprodukt und aktiver Metabolit des \\ Loratadins, ist ein neuer hochpotenter $\mathrm{H}_{1}$-Antagonist. Er greift in \\ die Akut- und Spätphase der allergischen Entzündungsreaktion \\ ein und wirkt zudem anti-obstruktiv.
}

A ntihistaminika der zweiten Generation wie Loratadin gehören zu den Arzneimitteln der ersten Wahl z.B. für die Behandlung der allergischen Rhinitis. Trotzdem bleibt ein Therapiedefizit: Sie lindern zwar Niesreiz und Hypersekretion, weniger jedoch die nasale $\mathrm{Ob}-$ struktion und die zugrunde liegende Entzündung. Doch gerade die Obstruktion beeinträchtigt die Lebensqualität von Patienten mit Rhinitis erheblich. Das neuentwickelte Desloratadin (Aerius $\left.^{\circledR}\right)$ kann diese Lücken in der Therapie schließen.

Der Pharmakologe Prof. Ulrich Borchard (Düsseldorf) stellt Desloratadin (DL) als ein hochpotentes, hochselektives Antihistaminikum vor, dessen Hemmwirkung auf H1-Rezeptoren die der anderen Antihistaminika deutlich übersteigt. So ist die H1-Rezeptoraffinität von DL etwa 200fach höher als die von Loratadin. Von besonderer Bedeutung ist die ausgeprägte antiinflammatorische Wirkung von DL, die auf einer

Freisetzungshemmung verschiedener Entzündungsmediatoren (z.B. Interleukine, Leukotriene, Prostaglandine) beruht. Zudem unterdrückt $\mathrm{DL}$ die Chemotaxis von Eosinophilen und ihre Adhäsion an Epithelzellen.

Die Resorption erfolgt rasch und ist von der Nahrung unabhängig. Bereits nach 30 min wird die antiallergische Wirkung deutlich messbar. Nach einer Stunde reduzieren sich Rhinitisbeschwerden wie Niesen, Juckreiz und laufende Nase um 50-70\%; der maximale Effekt wird nach ca. drei Stunden erreicht. Insbesondere vermindert Desloratadin auch die Obstruktion um $30 \%$, und zwar ohne Ermüdungseffekt, verkündet Borchard. Die Eliminationshalbwertszeit liegt bei $24 \mathrm{~h}$, so dass die einmal tägliche Gabe ausreicht.

\section{Borchard U.}

Desloratadin - pharmakologische Aspekte, Presse-Symposium der Essex Pharma, Oktober 2000.

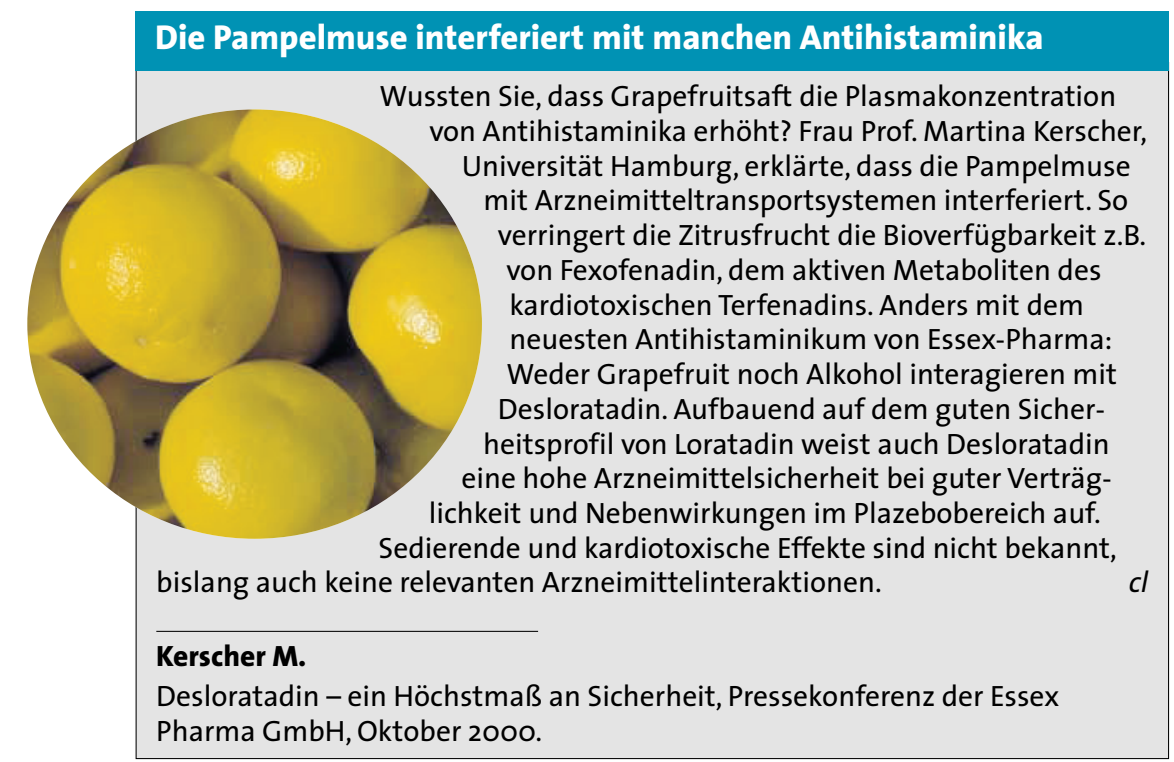

